\title{
ON THE RELATIVE GENERALIZED HAMMING WEIGHTS OF LINEAR CODES AND THEIR SUBCODES*
}

\author{
ZIHUI $\mathrm{LIU}^{\dagger}$, JIE WANG ${ }^{\ddagger}$, AND XIN-WEN WUई
}

\begin{abstract}
We first present an equivalent definition of relative generalized Hamming weights of a linear code and its subcodes, and we develop a method using finite projective geometry. Making use of the equivalent definition and the projective-geometry method, all of the relative generalized Hamming weights of a 3 -dimensional $q$-ary linear code and its subcodes will be determined.
\end{abstract}

Key words. generalized Hamming weights, relative generalized Hamming weights, support weight, relative difference sequence

AMS subject classification. 94B05

DOI. $10.1137 / 090770254$

1. Introduction and basic notations. Motivated by a cryptographical application of linear codes to the wire-tap channel of type II [1], generalized Hamming weights (GHWs) of a linear code have been defined and studied in [2]. GHWs not only characterize the algebraic structure of linear codes, but also have a lot of applications; for instance, application to trellis decoding of linear codes. In the past two decades, GHWs of linear codes have been extensively studied (see [3], [4], [5], [6], [7], and $[8])$.

Extending GHWs to a two-code format, the authors of [9] have introduced relative generalized Hamming weights (RGHWs) of a linear code $C$ and a subcode $C^{1}$ of $C$. When the subcode $C^{1}$ is zero, i.e., a code with zero vector as its unique codeword, the RGHWs of $C$ are exactly the traditional GHWs of $C$. Therefore, RGHWs are a generalization of GHWs. RGHWs are useful in analyzing the coordinated multiparty wire-tap channel of type II as discussed in [9]. More precisely, for the multiparty wiretap channel of type II with the coset coding scheme, the minimum uncertainty (also called equivocation) of the first parity's data bits to an adversary is characterized by the RGHWs (see Corollary 1 of [9]). Some upper and lower bounds on RGHWs have been given; conditions for achieving these bounds have been provided in [9]. Like the study of GHWs, the study of RGHWs is an interesting and important topic in algebraic coding theory. For a general linear code, it is difficult to determine all, or even part of, RGHWs, except for linear codes of small dimensions. In [10], all of the RGHWs of a 4-dimensional linear code with its 2-dimensional subcodes, and part of the RGHWs of a 4-dimensional linear code with its 1-dimensional subcodes, have been determined.

In this paper we first give an equivalent definition of the RGHWs. Based on the equivalent definition, we then develop a method using finite projective geometry,

${ }^{*}$ Received by the editors September 8, 2009; accepted for publication (in revised form) July 22, 2010; published electronically September 29, 2010. Supported by the Project Science Foundation of BIT.

http://www.siam.org/journals/sidma/24-4/77025.html

${ }^{\dagger}$ Corresponding author. Department of Mathematics, Beijing Institute of Technology, Beijing 100081, China (lzhui@bit.edu.cn).

${ }^{\ddagger}$ Department of Mathematics, Beijing University, Beijing 100081, China (wangj@math.pku.edu. $\mathrm{cn})$.

$\S$ School of Information and Communication Technology, Griffith University, Gold Coast, QLD 4222, Australia (wen@griffith.edu.au). 
motivated by the application of finite projective geometry to the study of traditional GHWs (see [4] and [8]). Making use of the projective geometry method, we will determine all of the RGHWs of a 3-dimensional linear code with its subcodes.

Let $C$ be an $[n, k]$ linear code and $\mathrm{J}$ be a subset of $\mathrm{I}=\{1, \ldots, n\}$. The subcode $C_{\mathrm{J}}$ of $C$ is defined as $C_{\mathrm{J}}=\left\{\left(c_{1}, \ldots, c_{n}\right) \in C: c_{t}=0\right.$ for $\left.t \notin \mathrm{J}\right\}$. For any subcode $D$ of $C$, the support of $D$, denoted by $\chi(D)$, is defined as the set of positions where not all the codewords of $D$ have zero coordinates. Define $w_{S}(D)=|\chi(D)|$.

Definition 1 (see [2]). Let $C$ be an $[n, k]$ linear code. For any $r, 1 \leq r \leq k$, the rth $G H W$ of $C$, denoted by $d_{r}(C)$ (or $d_{r}$ for short), is defined as

$$
d_{r}(C)=\min \left\{w_{S}(D): D \text { is an }[n, r] \text { subcode of } C\right\} .
$$

Note that $d_{1}(C)$ equals to the traditional minimum Hamming weight of $C$.

Definition 2 (see [9]). Let $C$ be an $[n, k]$ linear code and $C^{1}$ be a $k_{1}$-dimensional subcode of $C$. For any $j, 1 \leq j \leq k-k_{1}$, the $j$ th $R G H W$ of $C$ and $C^{1}$, denoted by $M_{j}\left(C, C^{1}\right)$ (or $M_{j}$ when $C$ and $C^{1}$ are clear), is defined as

$$
M_{j}\left(C, C^{1}\right)=\min \left\{|J|: \operatorname{dim}\left(C_{J}\right)-\operatorname{dim}\left(C^{1}{ }_{J}\right) \geq j\right\},
$$

or equivalently

$$
M_{j}\left(C, C^{1}\right)=\min \left\{|\mathrm{J}|: \operatorname{dim}\left(C_{\mathrm{J}}\right)-\operatorname{dim}\left(C_{\mathrm{J}}^{1}\right)=j\right\} .
$$

Obviously, when $C^{1}=\{0\}$, we have $M_{r}\left(C, C^{1}\right)=d_{r}(C)$, the rth $G H W$.

Definition 3. Let $M_{0}=0$. Then the relative difference sequence $(R D S)$ of the $[n, k]$ linear code $C$ with the $\left[n, k_{1}\right]$ subcode $C^{1}$ is the sequence $\left(i_{0}, i_{1}, \ldots, i_{k-k_{1}}\right)$, where

$$
i_{0}=n-M_{k-k_{1}}, i_{j}=M_{k-k_{1}-j+1}-M_{k-k_{1}-j}, 1 \leq j \leq k-k_{1} .
$$

Obviously, the $R D S\left(i_{0}, i_{1}, \ldots, i_{k-k_{1}}\right)$ and the sequence $\left(M_{1}, M_{2}, \ldots, M_{k-k_{1}}, n\right)$ can be determined from each other.

2. Finite projective geometry method. In this section, we give an equivalent definition of the RGHWs and some general results. We then develop a method using finite project geometry. These results and the projective geometry method will be critical to show our main results in next section. We first prove Lemma 1, part of which has been shown in [10].

Lemma 1. The RGHWs of $C$ and $C^{1}$ can also be described as follows:

$$
\begin{aligned}
M_{j}\left(C, C^{1}\right) & =\min \left\{w_{S}(D): D \text { is a subcode of } C, \operatorname{dim} D=j, D \cap C^{1}=\{0\}\right\} \\
& =\min \left\{w_{S}(D): D \text { is a subcode of } C, \operatorname{dim} D-\operatorname{dim} D \cap C^{1}=j\right\},
\end{aligned}
$$

where $0 \leq j \leq k-k_{1}$.

Proof. For $0 \leq j \leq k-k_{1}$, let

$$
\begin{aligned}
& r_{j}=\min \left\{w_{S}(D): D \text { is a subcode of } C, \operatorname{dim} D=j, D \cap C^{1}=\{0\}\right\}, \text { and } \\
& r_{j}^{\prime}=\min \left\{w_{S}(D): D \text { is a subcode of } C, \operatorname{dim} D-\operatorname{dim}\left(D \cap C^{1}\right)=j\right\} .
\end{aligned}
$$

We will show $r_{j}=r_{j}^{\prime}=M_{j}$.

It is obvious that $r_{j} \geq r_{j}^{\prime}$. We now show $r_{j} \leq r_{j}^{\prime}$. Let $D$ be a subcode of $C$, with $\operatorname{dim} D-\operatorname{dim}\left(D \cap C^{1}\right)=j$ and $r_{j}^{\prime}=w_{S}(D)$. Suppose $\boldsymbol{B}_{\mathbf{1}}$ is a generator matrix of $D \cap C^{1}$. It can be extended as a generator matrix $\boldsymbol{B}$ of $D$, as

$$
B=\left(\begin{array}{c}
B_{1} \\
B_{2}
\end{array}\right) \text {. }
$$

Copyright (c) by SIAM. Unauthorized reproduction of this article is prohibited. 
Then the subcode $D_{1}$ generated by $\boldsymbol{B}_{2}$ satisfies $\operatorname{dim} D_{1}=j, D_{1} \cap C^{1}=\{0\}$, and $w_{S}\left(D_{1}\right) \leq w_{S}(D)$, which implies that $r_{j} \leq r_{j}^{\prime}$.

Next we show $r_{j}^{\prime}=M_{j}$. By the definition of the RGHWs, we have $M_{0}=0$ and

$$
M_{j}\left(C, C^{1}\right)=\min \left\{|\mathrm{J}|: \operatorname{dim}\left(C_{\mathrm{J}}\right)-\operatorname{dim}\left(C_{\mathrm{J}}^{1}\right) \geq(=) j\right\}, \quad 1 \leq j \leq k-k_{1} .
$$

Since $C_{\mathrm{J}}$ is a subcode of $C$ and $C^{1}{ }_{\mathrm{J}}=C_{\mathrm{J}} \cap C^{1}$, we can get $r_{j}^{\prime} \leq M_{j}$.

Now we show $r_{j}^{\prime} \geq M_{j}$. Let $D$ be a subcode of $C$, with $r_{j}^{\prime}=w_{S}(D)$ and

$$
\operatorname{dim} D-\operatorname{dim} D \cap C^{1}=j .
$$

We have

$$
\begin{aligned}
& \operatorname{dim} C_{\chi(D)}-\operatorname{dim} C_{\chi(D)} \cap C^{1} \\
= & \operatorname{dim} C_{\chi(D)}-\operatorname{dim} C^{1} \chi(D) \\
= & \operatorname{dim}\left(C_{\chi(D)}+C^{1}\right)-\operatorname{dim} C^{1} \\
\geq & \operatorname{dim}\left(D+C^{1}\right)-\operatorname{dim} C^{1} \\
= & \operatorname{dim} D-\operatorname{dim} D \cap C^{1}=j .
\end{aligned}
$$

Therefore, $r_{j}^{\prime}=w_{S}(D)=|\chi(D)| \geq M_{j}$ by Definition 2 .

Let the RGHWs of $C$ and $C^{1}$ be $\left(M_{1}, M_{2}, \ldots, M_{k-k_{1}}\right)$. Let the GHWs of $C^{1}$ be $\left(d_{11}, d_{12}, \ldots, d_{1 k_{1}}\right)$ and the GHWs of $C$ be $\left(d_{1}, d_{2}, \ldots, d_{k}\right)$.

COROLlary 1. For $0 \leq t_{1} \leq k_{1}$ and $0 \leq t_{2} \leq k-k_{1}$, it holds that $d_{t_{1}+t_{2}} \leq$ $d_{1 t_{1}}+M_{t_{2}}$. In particular, $d_{1}=\min \left\{M_{1}, d_{11}\right\}$.

Proof. By Lemma 1, we can suppose that $M_{t_{2}}=w_{S}\left(D_{t_{2}}\right)$, where $D_{t_{2}} \cap C^{1}=$ $\{0\}$ and $\operatorname{dim} D_{t_{2}}=t_{2}$. Let $w_{S}\left(D_{1 t_{1}}\right)=d_{1 t_{1}}$, where $\operatorname{dim} D_{1 t_{1}}=t_{1}$ and $D_{1 t_{1}} \subset C^{1}$. Then $\operatorname{dim}\left(D_{1 t_{1}}+D_{t_{2}}\right)=t_{1}+t_{2}$. Now the claim of the corollary follows from Definition 1.

Let $\boldsymbol{u}=\left(u_{1}, u_{2}, \ldots, u_{k}\right) \in \operatorname{GF}(q)^{k}$ be a row vector (or the column vector $\left(u_{1}, u_{2}\right.$, $\left.\left.\ldots, u_{k}\right)^{T}\right)$. For $\mathrm{L} \subseteq\{1, \ldots, k\}$, define $\mathrm{P}_{\mathrm{L}}(\boldsymbol{u}) \in \mathrm{GF}(q)^{k}$ such that the $t$ th component of $\mathrm{P}_{\mathrm{L}}(\boldsymbol{u})$ is $u_{t}$ if $t \in \mathrm{L}$ and 0 if $t \notin \mathrm{L}$. An example is as follows: Let $\mathrm{L}=\{2,4\}$ and $\boldsymbol{u}=\left(u_{1}, u_{2}, u_{3}, u_{4}, u_{5}\right) \in \mathrm{GF}(q)^{5}$ (or the column vector $\left.\left(u_{1}, u_{2}, u_{3}, u_{4}, u_{5}\right)^{T}\right)$; then $\mathrm{P}_{\mathrm{L}}(\boldsymbol{u})=\left(0, u_{2}, 0, u_{4}, 0\right)\left(\right.$ or $\left.\mathrm{P}_{\mathrm{L}}(\boldsymbol{u})=\left(0, u_{2}, 0, u_{4}, 0\right)^{T}\right)$.

For a subset $U \subseteq \operatorname{GF}(q)^{k}$, we define $\mathrm{P}_{\mathrm{L}}(U)=\left\{\mathrm{P}_{\mathrm{L}}(\boldsymbol{u}): \boldsymbol{u} \in U\right\}$. Obviously, if $U$ is a subspace of $\operatorname{GF}(q)^{k}, \mathrm{P}_{\mathrm{L}}(U)$ is a subspace of $\mathrm{GF}(q)^{k}$.

Let $\boldsymbol{A}$ be a generator matrix of the $[n, k]$ linear code $C$. For any $\boldsymbol{u} \in \operatorname{GF}(q)^{k}$, the value of $\boldsymbol{u}$, denoted by $m(\boldsymbol{u})$, is defined as the number of occurrences of $\boldsymbol{u}$ as a column in $\boldsymbol{A}$. Define the value of a subset $U$ of $\operatorname{GF}(q)^{k}$ as follows:

$$
m(U)=\sum_{\boldsymbol{u} \in U} m(\boldsymbol{u})
$$

Lemma 2 (see [10]). Let

$$
\boldsymbol{A}=\left(\begin{array}{l}
\boldsymbol{A}_{k_{1} \times n} \\
\boldsymbol{A}_{\left(k-k_{1}\right) \times n}
\end{array}\right)
$$

be a generator matrix of $C$, where $\boldsymbol{A}_{\boldsymbol{k}_{1} \times \boldsymbol{n}}$ is a generator matrix for the $k_{1}$-dimensional subcode $C^{1}$. Then there is a one-to-one correspondence between the set of the $j$ dimensional subcodes $D$ satisfying $D \cap C^{1}=\{0\}$ and the set of the $(k-j)$-dimensional 
subspaces $U \subset G F(q)^{k}$ satisfying $\operatorname{dim}\left(P_{L}(U)\right)=k_{1}$ such that if $D$ corresponds to $U$, then $m(U)=n-w_{S}(D)$, where $1 \leq j \leq k-k_{1}$ and $L=\left\{1,2, \ldots, k_{1}\right\}$ represents the first $k_{1}$ coordinate positions of the vectors in $G F(q)^{k}$.

Let $C, C^{1}, D$, and $\boldsymbol{A}$ be as in Lemma 2. If $\boldsymbol{y}$ is a column of $\boldsymbol{A}$ and $\boldsymbol{x}=\alpha \boldsymbol{y}$ for some nonzero $\alpha \in \operatorname{GF}(q)$, then we may replace $\boldsymbol{y}$ by $\boldsymbol{x}$ without changing the support weight of the subcode $D$. Therefore, as in [4], we may view columns of $\boldsymbol{A}$ as points of the projective space $\mathrm{PG}(k-1, q)$. Then $m(\boldsymbol{x})$ means the number of occurrences of $\boldsymbol{x}$ in the columns of $\boldsymbol{A}$ for any point $\boldsymbol{x}$ of $\mathrm{PG}(k-1, q)$. Therefore, we can define a value function as

$$
m: \mathrm{PG}(k-1, q) \rightarrow \mathrm{N}=\{0,1,2, \ldots\} .
$$

For any point $\boldsymbol{x} \in \mathrm{PG}(k-1, q)$, we call $m(\boldsymbol{x})$ the value of $\boldsymbol{x}$. Correspondingly, $m(U)$ in Lemma 2 is called the value of the projective subspace $U$, when $U$ is a subspace, viewing the vectors in $U$ as points in the projective space $\mathrm{PG}(k-1, q)$.

By using the value function $m(\cdot)$, we can define a generator matrix and a code (up to equivalence) as follows. By Lemma 1, we can assume $M_{j}\left(C, C^{1}\right)=w_{S}\left(D^{*}\right)$ for some $j$-dimensional subcode $D^{*}$ satisfying $D^{*} \cap C^{1}=\{0\}$. Then, from Lemma $2, D^{*}$ corresponds to a $(k-j)$-dimensional subspace $U^{*} \subset \mathrm{GF}(q)^{k}$ satisfying $\operatorname{dim}\left(\mathrm{P}_{\mathrm{L}}\left(U^{*}\right)\right)=$ $k_{1}$ such that $m\left(U^{*}\right)=n-w_{S}\left(D^{*}\right)$. Now we consider $U^{*}$ as a $(k-j-1)$-dimensional projective subspace of $\mathrm{PG}(k-1, q)$ and still denote it as $U^{*}$. Then $\operatorname{dim}\left(\mathrm{P}_{\mathrm{L}}\left(U^{*}\right)\right)=$ $k_{1}-1$. Therefore, we have

(1)

$$
\begin{aligned}
& \max \left\{m(U): U \subseteq \mathrm{PG}(k-1, q) \text { with } \operatorname{dim}(U)=k-j-1 \text { and } \operatorname{dim}\left(\mathrm{P}_{\mathrm{L}}(U)\right)=k_{1}-1\right\} \\
&=m\left(U^{*}\right)=n-w_{S}\left(D^{*}\right)=n-M_{j}=\sum_{t=0}^{k-k_{1}-j} i_{t},
\end{aligned}
$$

where $0 \leq j \leq k-k_{1}$.

Remark. We now summarize the finite projective geometry method as follows: to construct a linear $k$-dimensional code $C$ and a $k_{1}$-dimensional subcode $C^{1}$ with the parameters $\left(M_{1}, M_{2}, \ldots, M_{k-k_{1}}, n\right)$, it is necessary to construct the value function $m(\cdot)$ satisfying $(1)$.

In the following section, we will employ this method to study the RGHWs of 3-dimensional linear codes and their subcodes.

3. RGHWs of a 3-dimensional linear code and its subcodes. In this section we study the RGHWs of a 3-dimensional linear code $C$ and its subcodes $C^{1}$. We will determine all the RGHWs of $C$ and $C^{1}$, making use of our method given in the previous section.

We call the 0-dimensional, 1-dimensional, and 2-dimensional subspaces of $\mathrm{PG}(k-$ $1, q)$ points, lines, and planes, respectively. We denote by $\overline{P Q}$ the line spanned by points $P$ and $Q$, and by $\widehat{P Q R}$ the plane spanned by points $P, Q$, and $R$.

Let $E=(1,0,0), F=(0,1,0)$, and $G=(0,0,1)$ denote the basis points in the projective plane $V=\mathrm{PG}(2, q)$ (see Figure 1 ).

Now let us consider a 3 -dimensional linear code $C$. Suppose $C^{1}$ is a 1 -dimensional subcode of $C$. By Lemma 2, there is a one-to-one correspondence between the set of all $j$-dimensional $(1 \leq j \leq 2)$ subcodes $D$ satisfying $D \cap C^{1}=\{0\}$ and the set of $(2-j)$-dimensional subspaces $U \subset \mathrm{PG}(2, q)$ satisfying $\operatorname{dim}\left(\mathrm{P}_{\mathrm{L}}(U)\right)=k_{1}-1=0$, where $\mathrm{L}=\{1\} \subset\{1,2,3\}$. It is clear that $\operatorname{dim}\left(\mathrm{P}_{\mathrm{L}}(U)\right)=0$ for $\mathrm{L}=\{1\}$ means $U \nsubseteq \overline{F G}$. Thus, from (1), the construction of $C$ and $C^{1}$ with the parameters $\left(M_{1}, M_{2}, n\right)$ is 


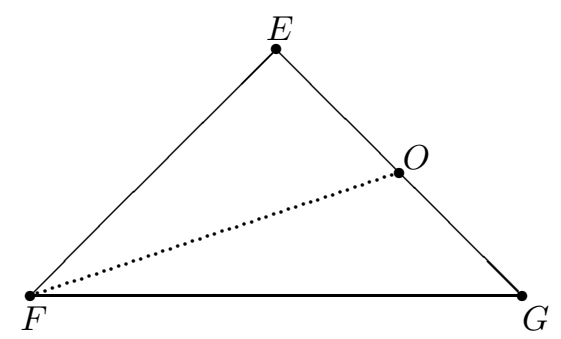

FIG. 1. Construction for the 3-dimensional codes.

equivalent to that of the value function $m(\cdot)$ such that

$$
\begin{aligned}
& \max \{m(p): p \text { is a point of } \mathrm{PG}(2, q) \text { and } p \notin \overline{F G}\}=i_{0}, \\
& \max \{m(l): l \text { is a line of } \mathrm{PG}(2, q) \text { and } l \neq \overline{F G}\}=i_{0}+i_{1}, \\
& m(V)=m(\mathrm{PG}(2, q))=i_{0}+i_{1}+i_{2} .
\end{aligned}
$$

Define

$$
\begin{aligned}
& \mathrm{MU}_{0}=\left\{p: m(p)=i_{0}, p \text { is a point and } p \notin \overline{F G}\right\} \\
& \mathrm{MU}_{1}=\left\{l: m(l)=i_{0}+i_{1}, l \text { is a line and } l \neq \overline{F G}\right\}
\end{aligned}
$$

By Lemma 1, we can assume that there exist a 1-dimensional subcode $D_{1}$ and a 2-dimensional subcode $D_{2}$ satisfying $w_{S}\left(D_{1}\right)=M_{1}, w_{S}\left(D_{2}\right)=M_{2}$, and $D_{1} \cap C^{1}=$ $D_{2} \cap C^{1}=\{0\}$. In what follows, we will distinguish the discussion into two cases according to whether $D_{1} \subset D_{2}$ or not.

Case A. $D_{1} \subset D_{2}$. Equivalently, we have to construct a value function $m(\cdot)$ satisfying $p \in l$ for a point $p \in \mathrm{MU}_{0}$ and a line $l \in \mathrm{MU}_{1}$. We call the RDS for this case, i.e., Case A, ARDS.

THEOREM 1. The sequence $\left(i_{0}, i_{1}, i_{2}\right)$ is an ARDS if and only if it satisfies $i_{0} \geq 1$, $i_{1} \geq 1$, and $1 \leq i_{2} \leq q i_{1}$.

Proof. $(\Rightarrow)$ Without loss of generality, we assume $E \in \mathrm{MU}_{0}$ and $\overline{E F} \in \mathrm{MU}_{1}$ (see Figure 1). Since the $q+1$ lines passing through the point $E$ in $V=\operatorname{PG}(2, q)$ are all the possible ones in the set $\mathrm{MU}_{1}$, so we have

$$
m(V)=m(\operatorname{PG}(2, q))=i_{0}+i_{1}+i_{2} \leq i_{0}+(q+1) i_{1},
$$

that is, $i_{2} \leq q i_{1}$.

$(\Leftarrow)$ We first construct $m(\cdot)$ for $i_{2}=q i_{1}$ as follows (see Figure 1):

$$
m(p)= \begin{cases}i_{0}, & \text { the point } p=E, \\ i_{1}, & \text { the point } p \in \text { the line } \overline{F G}, \\ 0, & \text { otherwise. }\end{cases}
$$

Obviously, we have $E \in \mathrm{MU}_{0}$ and $\overline{E F} \in \mathrm{MU}_{1}$. Note that $E \in \overline{E F}$. So, the value function $m(\cdot)$ satisfies the conditions stated in Case A. Thus, all the sequences $\left(i_{0}, i_{1}, i_{2}\right)$ such that $i_{0} \geq 1, i_{1} \geq 1$, and $i_{2}=q i_{1}$ are ARDSs.

If $i_{2}<q i_{1}$, we can decrease the values of the points $p \in \mathrm{PG}(2, q) \backslash \overline{E F}$ one by one until the value of $i_{2}$ is obtained.

Case B. We always have $D_{1} \nsubseteq D_{2}$ for any 1-dimensional subcode $D_{1}$ and any 2-dimensional subcode $D_{2}$ such that $D_{1} \cap C^{1}=D_{2} \cap C^{1}=\{0\}, w_{S}\left(D_{1}\right)=M_{1}\left(C, C^{1}\right)$, 
and $w_{S}\left(D_{2}\right)=M_{2}\left(C, C^{1}\right)$. Equivalently, we must find a value function $m(\cdot)$ such that $p \notin l$ for any point $p \in \mathrm{MU}_{0}$ and any line $l \in \mathrm{MU}_{1}$. We call the RDS for this case, i.e., Case B, BRDS.

THEOREM 2. (i) If the sequence $\left(i_{0}, i_{1}, i_{2}\right)$ is a BRDS, then $i_{0} \geq 1, i_{1} \geq 2, i_{2} \leq$ $q i_{1}-(q+1)$, and $i_{2} \geq i_{0}$.

(ii) All the sequences $\left(i_{0}, i_{1}, i_{2}\right)$ satisfying $i_{0} \geq 2\left(i_{0} \geq 3\right.$ if $\left.q=2\right), i_{2} \leq q i_{1}-(q+$ $1)$, and $i_{2} \geq i_{0}$ are BRDSs.

Proof. (i) Assume $\overline{F O} \in \mathrm{MU}_{1}$ and $E \in \mathrm{MU}_{0}$ (see Figure 1). Then, from the assumption that $\left(i_{0}, i_{1}, i_{2}\right)$ is a BRDS, we can show similarly to Theorem 1 that $i_{2} \leq q i_{1}-(q+1)$ and $i_{2} \geq i_{0}$. Observe that if $i_{1}=1$, then $i_{2} \leq q i_{1}-(q+1)<0$. Thus, $i_{1} \geq 2$.

To show (ii), let's construct $m(\cdot)$ for $i_{2}=q i_{1}-(q+1)$ such that $m(\cdot)$ satisfies the conditions stated in Case B.

Assume $i_{0}+1=q \alpha+\beta$ for $0 \leq \beta \leq q-1$. Choose $\beta$ points continuously on the line $\overline{F O}$ and denote them $O_{1}=O, O_{2}, \ldots, O_{\beta}$. Suppose $\overline{E O_{i}} \cap \overline{F G}=G_{i}$, where $1 \leq i \leq \beta$ (see Figure 1 ). Note that $G_{1}=G$. Then we can write $m(\cdot)$ as follows:

$$
m(p)= \begin{cases}i_{0}, & p=E, \\ i_{1}-1, & p=F, \\ \alpha+1, & p=O_{j}, 1 \leq j \leq \beta \\ \alpha, & p \in \overline{F O}, p \neq F, p \neq O_{j}, 1 \leq j \leq \beta, \\ i_{1}-2-\alpha, & p=G_{j}, 1 \leq j \leq \beta \\ i_{1}-1-\alpha, & p \in F G, p \neq F, p \neq G_{j}, 1 \leq j \leq \beta, \\ 0, & \text { otherwise. }\end{cases}
$$

Then $m(E)=i_{0}=\max \{m(p): p \notin \overline{F G}\}$ only if $i_{0}>\alpha+1$, which holds when $i_{0}>1+\frac{2}{q-1}$ or when $i_{0} \geq 2$ and $q \geq 4$.

Assume a line $l \neq \overline{F G}$. If $E \in l$ and $O_{j} \in l$ for some $1 \leq j \leq \beta$, then $m(l)=$ $m(E)+m\left(O_{j}\right)+m\left(G_{j}\right)=i_{0}+(\alpha+1)+\left(i_{1}-2-\alpha\right)=i_{0}+i_{1}-1<i_{0}+i_{1}$. In addition, $m(\overline{E F})=m(E)+m(F)=i_{0}+i_{1}-1<i_{0}+i_{1}$.

For other lines $l$ satisfying $l \neq \overline{F G}$ and $E \in l$ we can similarly obtain $m(l)=$ $i_{0}+i_{1}-1<i_{0}+i_{1}$.

If $l \neq \overline{F G}, F \notin l$, and $E \notin l$, then $m(l) \leq\left(i_{1}-1-\alpha\right)+(\alpha+1)=i_{1}<i_{0}+i_{1}$. For the lines $l$ such that $E \notin l, F \in l$, and $l \neq \overline{F O}$, we have $m(l)=m(F)=i_{1}-1<$ $i_{0}+i_{1}$. In addition, $m(\overline{F O})=m(F)+(q-\beta) \alpha+\beta(\alpha+1)=i_{0}+i_{1}$. So we can obtain $m(\overline{F O})=\max \{m(l): l \neq \overline{F G}\}=i_{0}+i_{1}$.

The arguments above show that $m(\cdot)$ satisfies the conditions stated in Case B. So all the sequences $\left(i_{0}, i_{1}, i_{2}\right)$ such that $i_{0} \geq 2, i_{2}=q i_{1}-(q+1)$, and $i_{2} \geq i_{0}$ are BRDSs when $q \geq 4$.

There remain the sequences $\left(i_{0}, i_{1}, i_{2}\right)$ such that $i_{2} \leq q i_{1}-(q+1), i_{2} \geq i_{0}$, $i_{0}=1$ for $q \geq 4,1 \leq i_{0} \leq 2$ for $q=3$, and $1 \leq i_{0} \leq 3$ for $q=2$ to cope with.

We first show that the sequences $i_{0}=1, i_{2} \leq q i_{1}-(q+1)$, and $i_{2} \geq i_{0}$ for any $q \geq 2$ are not BRDSs.

On the contrary, assume there exists a sequence such that $i_{0}=1, i_{2} \leq q i_{1}-(q+$ 1 ), and $i_{2} \geq i_{0}$, which is a BRDS. Then there exists a value function $m(\cdot)$ satisfying the conditions stated in Case B. We suppose $m\left(p_{0}\right)=i_{0}=1=\max \{m(p): p \notin \overline{F G}\}$ and $m\left(l_{0}\right)=i_{0}+i_{1}=i_{1}+1=\max \{m(l): l \neq \overline{F G}\}$ (see Figure 1). Assume $l_{0} \cap \overline{F G}=p^{\prime}$. Since any point $p \in \mathrm{MU}_{0}$ is not on the line $l_{0}, m(p)=0$ for any $p \in l_{0} \backslash p^{\prime}$. So $m\left(l_{0}\right)=m\left(p^{\prime}\right)=i_{0}+i_{1}$. Then $m\left(\overline{p_{0} p^{\prime}}\right) \geq m\left(p_{0}\right)+m\left(p^{\prime}\right)=2 i_{0}+i_{1}>$ $\max \{m(l): l \neq \overline{F G}\}=i_{0}+i_{1}$, which is a contradiction. Thus, we can conclude that 
the sequences $i_{0}=1, i_{2} \leq q i_{1}-(q+1)$ and $i_{2} \geq i_{0}$ for any $q \geq 2$ are not BRDSs.

Note that $q \mid\left(i_{0}+1\right)$ when $i_{0}=2$ for $q=3$ and $i_{0}=3$ for $q=2$. So $m(\cdot)$ in (2) becomes the following one:

$$
m(p)= \begin{cases}i_{0}, & p=E, \\ i_{1}-1, & p=F, \\ \alpha, & p \in \overline{F O}, p \neq F, \\ i_{1}-1-\alpha, & p \in \overline{F G}, p \neq F, \\ 0, & \text { otherwise. }\end{cases}
$$

Then it is not difficult to check that the sequences $\left(i_{0}, i_{1}, i_{2}\right)$ such that $i_{2}=q i_{1}-(q+$ 1), $i_{2} \geq i_{0}, i_{0}=2$ for $q=3$ and $i_{0}=3$ for $q=2$ are all BRDSs.

Lastly we show that the sequences $i_{2} \leq q i_{1}-(q+1), i_{2} \geq i_{0}$, and $i_{0}=2$ for $q=2$ are not BRDSs. On the contrary, assume that there exists a sequence $\left(i_{0}, i_{1}, i_{2}\right)$ which is a BRDS. Then there is a corresponding $m(\cdot)$. Assume $p_{0} \in \mathrm{MU}_{0}$ and $l_{0} \in \mathrm{MU}_{1}$. Suppose $l_{0} \cap \overline{F G}=p^{\prime}$ and denote the other two points on the line $l_{0}$ as $p_{1}$ and $p_{2}$. Then $m\left(p_{1}\right) \leq i_{0}-1=1$ and $m\left(p_{2}\right) \leq i_{0}-1=1$ from the assumption that $\left(i_{0}, i_{1}, i_{2}\right)$ is a BRDS. So, $m\left(p^{\prime}\right)=m\left(l_{0}\right)-m\left(p_{1}\right)-m\left(p_{2}\right) \geq\left(i_{0}+i_{1}\right)-1-1=i_{1}$. Then we have $m\left(\overline{p_{0} p^{\prime}}\right) \geq m\left(p_{0}\right)+m\left(p^{\prime}\right) \geq i_{0}+i_{1}$. Since $\max \{m(l): l \neq \overline{F G}\}=i_{0}+i_{1}$ and $\overline{p_{0} p^{\prime}} \neq \overline{F G}$, we have $m\left(\overline{p_{0} p^{\prime}}\right)=i_{0}+i_{1}$, which contradicts the assumption that $\left(i_{0}, i_{1}, i_{2}\right)$ is a BRDS. Therefore, the sequences $i_{2} \leq q i_{1}-(q+1), i_{2} \geq i_{0}$, and $i_{0}=2$ for $q=2$ are not BRDSs.

If $i_{2}<q i_{1}-(q+1)$, it is necessary to decrease the values of the points $p \in$ $P G(2, q) \backslash(\overline{F O} \bigcup\{E\})$ one by one until $i_{2}=i_{0}$.

Now we consider the RGHWs of a 3 -dimensional linear code $C$ and a 2-dimensional subcode $C^{1}$ of $C$. The RDS is $\left(i_{0}, i_{1}\right)$, where $i_{0} \geq 2$ and $i_{1} \geq 1$. By Lemma 2 we can express the value function $m(\cdot)$ as follows (see Figure 1 ):

$$
\max \{m(l): G \notin l\}=i_{0}, m(V)=i_{0}+i_{1} .
$$

Theorem 3. For any $i_{0} \geq 2, i_{1} \geq 1$, there exist a 3 -dimensional linear code $C$ and a 2-dimensional subcode $C^{1}$ of $C$ such that the $R D S$ is $\left(i_{0}, i_{1}\right)$.

Proof. Assume $i_{0}=(q+1) \alpha+\beta$ for $0 \leq \beta \leq q$, and denote the $q+1$ points on the line $\overline{E F}$ by $E_{0}=E, E_{1}, \ldots, E_{q}=F$, respectively. We then construct $m(\cdot)$ (see Figure 1) as follows:

$$
m(p)= \begin{cases}\alpha, & p=E_{i} \text { for } 0 \leq i \leq q-\beta, \\ \alpha+1, & p=E_{i} \text { for } q-\beta+1 \leq i \leq q, \\ i_{1}, & p=G, \\ 0, & \text { otherwise }\end{cases}
$$

Obviously, we have

$$
m(\overline{E F})=\max \{m(l): G \notin l\}=i_{0} \text { and } m(V)=i_{0}+i_{1} .
$$

Thus, any sequence $\left(i_{0}, i_{1}\right)$ satisfying $i_{0} \geq 2$ and $i_{1} \geq 1$ is an RDS.

Acknowledgments. The authors would like to thank the anonymous reviewers and Associate Editor, Prof. Ron Roth, for their valuable comments that helped to improve this paper.

\section{REFERENCES}

[1] L. H. Ozarow and A. D. Wyner, Wire-tap channel II, AT\&T Bell Laboratories Tech. J., 63 (1984), pp. 2135-2157. 
[2] V. K. WeI, Generalized Hamming weights for linear codes, IEEE Trans. Inform. Theory, 37 (1991), pp. 1412-1418.

[3] T. Helleseth, T. Kløve, and Ø. Ytrehus, Generalized Hamming weights of linear codes, IEEE Trans. Inform. Theory, 38 (1992), pp. 1133-1140.

[4] W. D. Chen And T. Kløve, The weight hierarchies of q-ary codes of dimension 4, IEEE Trans. Inform. Theory, 42 (1996), pp. 2265-2272.

[5] W. D. Chen And T. Kløve, Weight hierarchies of extremal non-chain binary codes of dimension 4, IEEE Trans. Inform. Theory, 45 (1999), pp. 276-281.

[6] W. D. Chen and T. Kløve, Weight hierarchies of linear codes of dimension 3, J. Statist. Plann. Inference, 94 (2001), pp. 167-179.

[7] W. D. Chen And T. Kløve, Finite projective geometries and classification of the weight hierarchies of codes (I), Acta Math., 20 (2004), pp. 333-348.

[8] M. A. Tsfasman and S. Vladuts, Geometric approach to higher weights, IEEE Trans. Inform. Theory, 41 (1995), pp. 1564-1588.

[9] Y. Luo, M. Chaichana, A. J. Han Vinck, and K. F. Chen, Some new characters on the wire-tap channel of type II, IEEE Trans. Inform. Theory, 51 (2005), pp. 1222-1229.

[10] Z. H. Liu, W. D. Chen, AND Y. Luo, The relative generalized Hamming weight of linear q-ary codes and their subcodes, Des. Codes Cryptogr., 48 (2008), pp. 111-123.

Copyright (c) by SIAM. Unauthorized reproduction of this article is prohibited. 